\title{
Testing the fuel-mediated hypothesis: maternal insulin resistance and glucose mediate the association between maternal and neonatal adiposity, the Healthy Start study
}

\author{
Allison L. B. Shapiro • Sarah J. Schmiege • \\ John T. Brinton • Deborah Glueck • Tessa L. Crume • \\ Jacob E. Friedman • Dana Dabelea
}

Received: 23 September 2014 / Accepted: 9 January 2015 / Published online: 28 January 2015

(C) Springer-Verlag Berlin Heidelberg 2015

\begin{abstract}
Aims/hypothesis In women who are overweight or obese before or during pregnancy there is an associated risk of increased fetal growth and higher birthweight. The metabolic phenotype of the overweight/obese pregnant woman, characterised by higher than normal insulin resistance (IR) and increased circulating fuels, suggests a mechanism resulting in fetal overnutrition and subsequent increased adiposity. We tested the fuel-mediated hypothesis in an observational pre-birth cohort of 951 mother-offspring pairs, the Healthy Start study.

Methods We conducted a path analysis to estimate the simultaneous effects of maternal IR and maternal fuels (fasting glucose, triacylglycerol [TG] and NEFA levels) in late pregnancy in mediating the relationship between maternal pre-pregnancy $\mathrm{BMI}$ and neonatal adiposity (per cent fat mass [\%FM]).
\end{abstract}

Electronic supplementary material The online version of this article (doi:10.1007/s00125-015-3505-z) contains peer-reviewed but unedited supplementary material, which is available to authorised users.

A. L. B. Shapiro · T. L. Crume • D. Dabelea $(\bowtie)$

Department of Epidemiology, Colorado School of Public Health, Campus Box B119, 13001 East 17th Ave, Room W3110,

Aurora, CO 80045, USA

e-mail: dana.dabelea@ucdenver.edu

S. J. Schmiege $\cdot$ J. T. Brinton $\cdot$ D. Glueck

Department of Biostatistics and Bioinformatics, Colorado School of Public Health, University of Colorado, Denver, CO, USA

\section{J. E. Friedman}

Department of Pediatrics, School of Medicine, University of

Colorado, Denver, CO, USA

J. E. Friedman

Department of Biochemistry and Molecular Genetics, School of Medicine, University of Colorado, Denver, CO, USA
Results The total effect of maternal BMI on neonatal \%FM was significant (total effect $0.16,95 \%$ CI $0.08,0.22$, $p<0.001)$. The mediated path including maternal IR and glucose levels together accounted for $21 \%(p<0.01)$ of the total effect of maternal BMI on neonatal \%FM while the mediating effects of all other fuels were non-significant.

Conclusions/interpretation Using a novel application of path analysis our data implicate maternal IR and glucose levels as important mediators of the association between maternal and infant adiposity.

Keywords Epidemiology $\cdot$ Metabolic syndrome $\cdot$ Pregnancy

$\begin{array}{ll}\text { Abbreviations } \\ \text { FM } & \text { Fat mass } \\ \text { FFM } & \text { Fat-free mass } \\ \text { HAPO } & \text { Hyperglycemia and Adverse Pregnancy Outcome } \\ \text { IR } & \text { Insulin resistance } \\ \text { TG } & \text { Triacylglycerol } \\ \% \text { FM } & \text { Per cent fat mass }\end{array}$

\section{Introduction}

The perinatal period is a critical window for future development. Exposure to maternal obesity during pregnancy is a risk factor for increased fetal growth and adiposity $[1,2]$ as well as susceptibility to obesity and type 2 diabetes later in life $[3,4]$. Due to the long-term impact of the intrauterine environment, a better understanding of potential mechanisms is important to enable the design of primordial prevention programmes targeting women of reproductive age. 
Increased maternal BMI before pregnancy is linked to numerous negative metabolic outcomes for which the mechanisms of action remain poorly understood. It has been suggested that these associations are due in part to specific intrauterine effects resulting from the altered metabolic state of pregnant overweight and obese mothers, including increased insulin resistance (IR) and/or elevated maternal fuels (glucose, lipids), leading to fetal overnutrition $[5,6]$.

Using a multiple-mediator approach within a path analytic framework we sought to investigate whether maternal IR or glucose, triacylglycerol (TG) or NEFA levels in late pregnancy mediate the association between maternal pre-pregnancy BMI and neonatal adiposity, as measured by per cent fat mass (\%FM), using data from a large pre-birth cohort study in Colorado.

\section{Methods}

The Healthy Start study is an observational pre-birth cohort study of ethnically diverse mothers investigating the impact of pregnancy factors on infant body composition and metabolic health. Pregnant women aged 16 years and over with a gestational age less than 24 weeks were recruited from the University obstetrics clinics during 2010-2014. Women were excluded if they reported having pre-existing type 1 or type 2 diabetes, a prior premature birth or fetal death, asthma with steroid management, serious psychiatric illness or a current multiple pregnancy. All women provided written informed consent and the study was approved by the Colorado Multiple Institutional Review Board.

Women were invited to participate in two pregnancy research visits, at $<24$ weeks of gestation and at 24-32 weeks. Maternal pre-pregnancy BMI was calculated using measured height and pre-pregnancy weight obtained from medical records $(81.5 \%)$ or self-reported (12.5\%). Demographic, behavioural and dietary surveys were administered at each visit. A third visit occurred in the hospital, after delivery. Neonatal body composition, fat mass (FM) and fat-free mass (FFM) were measured within $48 \mathrm{~h}$ after delivery and \%FM was calculated by dividing FM (g) by the total body mass. Blood samples collected at the second visit were analysed by the University of Colorado Hospital Clinical and Translational Science Centers Core Laboratory. To estimate IR in pregnancy, the HOMA-IR equation was used.

Statistical analyses Tests for trend and Cochran MantelHaenszel tests were used to compare maternal and infant characteristics across maternal pre-pregnancy BMI categories (normal weight, $\mathrm{BMI}<25 \mathrm{~kg} / \mathrm{m}^{2}$; overweight, $25 \mathrm{~kg} / \mathrm{m}^{2} \leq$ BMI $<30 \mathrm{~kg} / \mathrm{m}^{2}$; obese, BMI $\geq 30 \mathrm{~kg} / \mathrm{m}^{2}$ ) (Table 1).

The main exposure of interest was maternal pre-pregnancy BMI and the main outcome was neonatal \%FM. Hypothesised mediators included IR, maternal fasting glucose, TG and total
NEFA levels. When necessary, values were log-transformed. An a priori list of covariates was assessed and were included in the final model if they were significantly $(p<0.05)$ associated with $\%$ FM. The final model included infant sex, gravidity and gestational age at birth.

Path model A multiple-mediator path model using robust maximum likelihood estimation was implemented. Analyses were completed using M-plus 6.0 (Muthén \& Muthén, Los Angeles, CA, USA). The disturbance terms of maternal glucose, TG and NEFA were allowed to correlate in the model. Standardised $\beta$ coefficients for the total effect of maternal pre-pregnancy BMI on neonatal $\% \mathrm{FM}$ were generated. Standardised $\beta$ coefficients for the direct and indirect effect of maternal pre-pregnancy BMI on neonatal $\% \mathrm{FM}$, and effects between pathway variables were also generated. Indirect effects were calculated with the product of coefficients method using Sobel's standard error. Additional information about the statistical model is provided in the electronic supplementary material (ESM) Methods.

\section{Results}

A total of 951 mother-offspring pairs with complete data were included. Table 1 displays maternal and infant characteristics by maternal pre-pregnancy BMI category. All maternal characteristics, except prevalence of smoking and age at delivery, differed across pre-pregnancy BMI categories. Maternal IR, glucose and NEFA followed a linear increasing pattern with increasing pre-pregnancy BMI $(p<0.01)$. Infant outcomes of birthweight, FM and \%FM, though not FFM, also increased across the pre-pregnancy BMI categories $(p<0.01)$.

Figure 1 displays the pathways tested with the respective standardised $\beta$ coefficients representing the total and direct effects of pre-pregnancy BMI on neonatal \%FM, as well as the relationships between each variable within the pathways explored. The total effect of maternal pre-pregnancy BMI on neonatal $\% \mathrm{FM}$ at birth was significant (total effect $=0.16,95 \%$ CI $0.08,0.22, p<0.001)$. For each $\mathrm{kg} / \mathrm{m}^{2}$ increase in prepregnancy BMI, neonatal \%FM increased by 0.16 percentage points. The direct effect of pre-pregnancy BMI on \%FM was attenuated but remained significant after including the mediators in the model (direct effect $=0.11,95 \%$ CI $0.04,0.18$, $p<0.01$ ).

The pathway leading from pre-pregnancy BMI to neonatal $\% \mathrm{FM}$ through increased maternal IR and increased glucose levels was significant (indirect effect $=0.03,95 \%$ CI 0.01 , $0.05, p<0.01$ ), accounting for $21 \%$ of the total effect of maternal BMI on neonatal \%FM. The two alternative fuelmediated pathways, testing the effect of maternal prepregnancy BMI on neonatal \%FM through increased IR and NEFA levels and increased IR and TG levels, were non- 
Table 1 Characteristics of study participants by pre-pregnancy BMI category

\begin{tabular}{|c|c|c|c|c|}
\hline \multirow[t]{2}{*}{ Characteristic } & \multicolumn{3}{|c|}{ Pre-pregnancy BMI category ${ }^{a}$} & \multirow[t]{2}{*}{$p$ value $^{\mathrm{b}}$} \\
\hline & Normal $(n=516)$ & Overweight $(n=244)$ & Obese $(n=191)$ & \\
\hline \multicolumn{5}{|l|}{ Mother } \\
\hline Age at delivery, years, mean (SD) & $27.7(6.1)$ & $28.0(6.2)$ & $27.8(5.8)$ & 0.86 \\
\hline Race/ethnicity, $n(\%)$ & & & & $<0.01$ \\
\hline Non-Hispanic white & $328(63.6)$ & $117(48.0)$ & $74(38.7)$ & \\
\hline Hispanic & $79(15.3)$ & $81(33.2)$ & $62(32.5)$ & \\
\hline Non-Hispanic black & $72(13.9)$ & $35(14.3)$ & $45(23.6)$ & \\
\hline Other & $37(7.2)$ & $11(4.5)$ & $10(5.2)$ & \\
\hline Gravidity, mean (SD) & $1.0(1.3)$ & $1.6(1.7)$ & $1.9(1.7)$ & $<0.01$ \\
\hline IOM recommended GWG, $n(\%)$ & & & & $<0.01$ \\
\hline Inadequate & $134(26.0)$ & $33(13.5)$ & $42(22.0)$ & \\
\hline Adequate & $167(32.4)$ & $57(23.4)$ & $39(20.4)$ & \\
\hline Excessive & $214(41.6)$ & $154(63.1)$ & $110(57.6)$ & \\
\hline Gestation length, weeks, mean (SD) & $39.6(1.1)$ & $39.6(1.1)$ & $39.3(1.1)$ & $<0.01$ \\
\hline Caesarean section, $n(\%)$ & $91(17.8)$ & $57(23.5)$ & $56(30.3)$ & $<0.01$ \\
\hline Smoking in pregnancy, $n(\%)$ & $44(8.5)$ & $17(7.0)$ & $25(13.1)$ & 0.07 \\
\hline Household income per year, $n(\%)$ & & & & $<0.01$ \\
\hline$<\$ 20,000$ & $54(12.9)$ & $36(18.1)$ & $52(34.7)$ & \\
\hline$\$ 20,000-\$ 40,000$ & $59(14.1)$ & $37(18.6)$ & $39(26.0)$ & \\
\hline$\$ 40,000-\$ 70,000$ & $95(22.7)$ & $53(26.6)$ & $30(20.0)$ & \\
\hline$>\$ 70,000$ & $211(50.4)$ & $73(36.7)$ & $29(19.3)$ & \\
\hline HOMA-IR, mean (SD) & $2.9(3.3)$ & $4.1(6.0)$ & $5.2(4.6)$ & $<0.01$ \\
\hline Glucose, mmol/l, mean (SD) & $4.2(0.4)$ & $4.4(0.4)$ & $4.5(0.6)$ & $<0.01$ \\
\hline $\mathrm{TG}, \mathrm{mmol} / \mathrm{l}$, mean $(\mathrm{SD})$ & $1.7(0.6)$ & $1.9(0.8)$ & $1.8(0.7)$ & 0.01 \\
\hline NEFA, mmol/l, mean (SD) & $0.35(0.15)$ & $0.38(0.15)$ & $0.43(0.16)$ & $<0.01$ \\
\hline \multicolumn{5}{|l|}{ Offspring } \\
\hline Female sex, $n(\%)$ & $263(51.0)$ & $117(48.0)$ & $81(42.4)$ & 0.04 \\
\hline Birthweight, g, mean (SD) & $3,245.0(420.3)$ & $3,322.9(456.8)$ & $3,325.1(441.1)$ & 0.03 \\
\hline FM, g, mean (SD) & $280.5(143.2)$ & $306.7(151.9)$ & $332.6(159.6)$ & $<0.01$ \\
\hline FFM, g, mean (SD) & $2,830.7(324.8)$ & $2,877.0(366.7)$ & $2,853.3(333.8)$ & 0.43 \\
\hline$\%$ FM, mean (SD) & $8.8(3.8)$ & $9.4(3.9)$ & $10.1(4.0)$ & $<0.01$ \\
\hline
\end{tabular}

${ }^{\mathrm{a}}$ Sum of column per cent values may not equal $100 \%$ due to missing data

${ }^{\mathrm{b}} p$ values were generated using trend analysis for continuous variables; Cochran Mantel-Haenszel tests were used for categorical variables GWG, gestational weight gain; IOM, Institute of Medicine

significant. The results were unchanged after removing data for the 35 women who were diagnosed with gestational diabetes.

\section{Discussion}

Maternal pre-pregnancy BMI was associated with increased neonatal adiposity across the entire range of BMI levels. Importantly and novel, our data suggest that increasing maternal IR and glucose levels, even within normal range, are responsible for $21 \%$ of the association between pre-pregnancy BMI and neonatal adiposity, with no significant contribution from other fuels. Our results provide further evidence that the relationship between maternal and offspring adiposity is due, in part, to specific fuel-mediated intrauterine effects on infant FM.

A continuous association between maternal glucose in late pregnancy and offspring birthweight/per cent body fat greater than 90th percentiles was also shown by the Hyperglycemia and Adverse Pregnancy Outcome (HAPO) study [7]. Our findings are consistent with those of the HAPO study and those of a recent study in a multi-ethnic Asian cohort in which a significant continuous effect of maternal glucose during pregnancy on neonatal adiposity was reported [8]. 
Fig. 1 Revised path model depicting tested mediated pathways between pre-pregnancy BMI, HOMA-IR, maternal TG, glucose and NEFA, and neonatal $\%$ FM at birth (solid arrows). Covariates were infant sex, gravidity and gestational age at birth. Dashed single-headed arrows indicate other associations not initially hypothesised in the model, but were included based on improving model fit and supported by theory. Dashed double-headed arrows indicate that the variable disturbance terms were specified as correlated in the model. ${ }^{*} p<0.05$

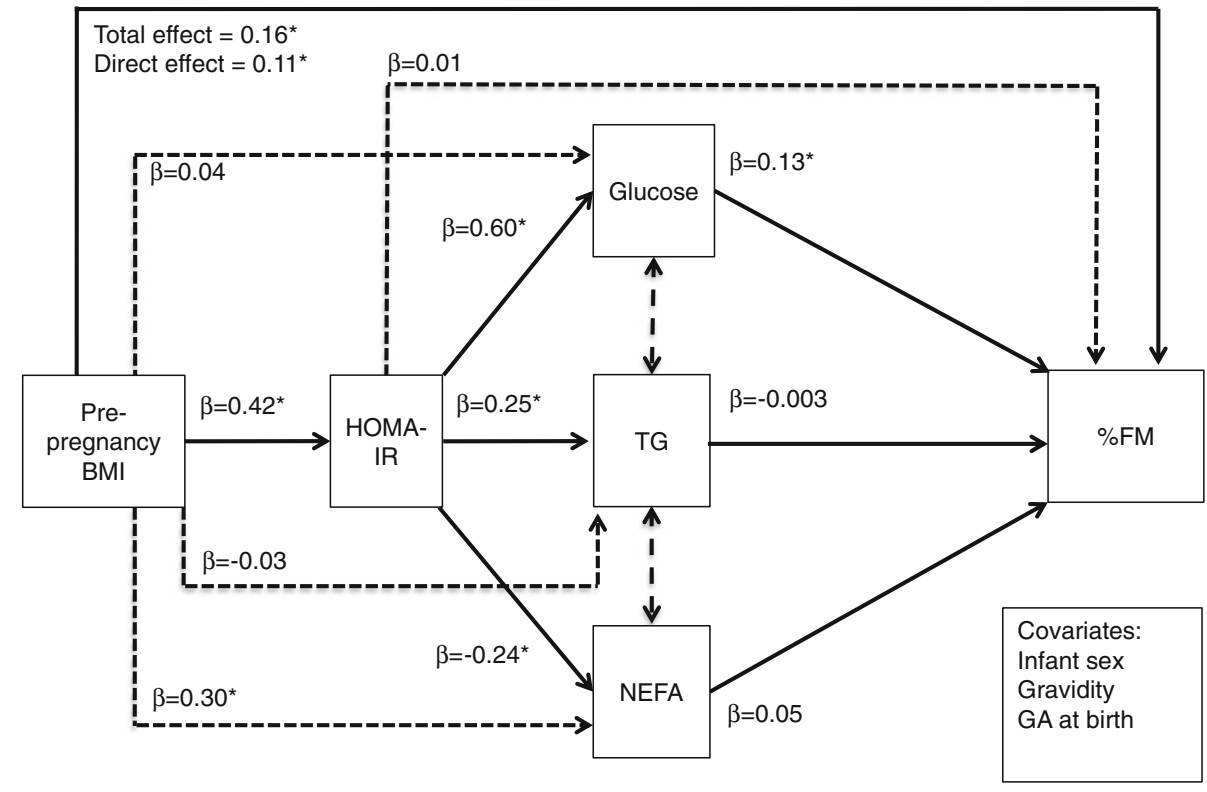

Interestingly, while maternal pre-pregnancy BMI correlated significantly with maternal plasma TG and NEFA levels (data not shown), in the final pathway model these fuels did not act as mediators of the relationship of interest. Another study in Colorado, conducted under controlled dietary conditions, found that maternal TG levels measured early in pregnancy correlated strongly with neonatal FM at birth [5]. However, the authors of that study did not control for other fuels (potential confounders) in their analysis. Similar to our results, the Norwegian STORK study (an observational, prospective study on the determinants of fetal growth and birthweight in healthy pregnancies) found that maternal BMI and fasting glucose were the only significant contributors to infant \%FM in multivariable models, while no other maternal fuels were associated with infant adiposity [9].

We found that maternal IR was negatively associated with late-pregnancy NEFA levels. This is contrary to our finding with TG as we expected both fuels to follow a similar increasing trend. With increasing IR, fasting-induced lipolysis of stored TG increases NEFA release. The metabolic fate of NEFA released from maternal adipose tissue stores includes flux across the placenta, oxidation in maternal tissues and, potentially, re-esterification with glucose to TG in the placenta [10]. Thus, a possible explanation for the unexpected inverse relationship between IR and NEFA levels is that increased IR may lead to increased NEFA uptake by the placenta or increased lipid synthesis in the maternal liver. As a result, we see a positive association between IR and TG but an inverse relationship between IR and NEFA levels.

Although we found that maternal pre-pregnancy BMI was significantly associated with neonatal adiposity, the glucosemediated pathway accounted for only $21 \%$ of the association, leaving $79 \%$ of this relationship unexplained by fuel-mediated pathways. Future research should examine more complex mediating models including, in addition to the fuel-mediated pathway, other likely mechanisms, such as epigenetic modifications, while carefully accounting for genetic susceptibility.

Our study has some limitations and several strengths. As with all observational studies, there is potential for residual confounding by unmeasured variables. Further, nondifferential measurement error in our mediators could contribute to an underestimation of our indirect effect. However, we do not believe this error to be large. While the epidemiology literature has many examples of single-mediation analysis, no studies have investigated multiple maternal factors together as potential mediators of the association between pre-pregnancy BMI and neonatal body composition.

In conclusion, our study suggests that part of the association between maternal and offspring adiposity is due to specific intrauterine effects-increased maternal IR and fasting glucose levels, leading to neonatal FM accretion. These effects are seen over the entire range of maternal BMI, IR and blood glucose levels in pregnancy. Our findings may generate further hypotheses regarding early-life programming of obesity.

Contribution statement ALBS was responsible for conception of the analytical approach, analysing the data, drafting the initial manuscript and editing each draft. SJS provided substantial statistical guidance, helped in drafting and revising the methods for the path analysis as well as the interpretation of results. JTB and DG contributed to the statistical analysis and contributed to data management and revisions of each draft. TLC contributed substantially to acquisition of data and assisted in revising each manuscript draft critically for intellectual content. JEF contributed scientific input and critical revisions to each manuscript draft to improve intellectual and scientific interpretation. DD provided substantial contribution to the conception and design of the Healthy Start study and this 
analysis and revised each draft of the manuscript. All authors approved the final version of the manuscript to be published. DD is responsible for the integrity of the work as a whole.

Acknowledgements We would like to thank M. Martinez (the Healthy Start Study Project Coordinator, Colorado School of Public Health, University of Colorado Denver, CO, USA) and the Healthy Start team for their hard work and dedication. We would also like to thank L. Barbour (Department of Endocrinology, University of Colorado, Denver, CO, USA) for her scientific input regarding metabolism in normal and overweight/obese pregnancy.

Funding The Healthy Start study is supported by a RO1 grant from the National Institute of Diabetes and Digestive and Kidney Disease (NIDDK) (RO1 no. DK076648 to principal investigator, DD).

Duality of interest The authors declare that there is no duality of interest associated with this manuscript.

\section{References}

1. Sewell MF, Huston-Presley L, Super DM, Catalano P (2006) Increased neonatal fat mass, not lean body mass, is associated with maternal obesity. Am J Obstet Gynecol 195:1100-1103

2. Hull HR, Dinger MK, Knehans AW, Thompson DM, Fields DA (2008) Impact of maternal body mass index on neonate birthweight and body composition. Am J Obstet Gynecol 198:416.e1-e6
3. Boney CM, Verma A, Tucker R, Vohr BR (2005) Metabolic syndrome in childhood: association with birth weight, maternal obesity, and gestational diabetes mellitus. Pediatrics 115:e290-e296

4. Hochner H, Friedlander Y, Calderon-Margalit R et al (2012) Associations of maternal prepregnancy body mass index and gestational weight gain with adult offspring cardiometabolic risk factors: the Jerusalem Perinatal Family Follow-up Study. Circulation 125:1381-1389

5. Harmon KA, Gerard L, Jensen DR et al (2011) Continuous glucose profiles in obese and normal-weight pregnant women on a controlled diet: metabolic determinants of fetal growth. Diabetes Care 34:2198-2204

6. Endo S, Maeda K, Suto M et al (2006) Differences in insulin sensitivity in pregnant women with overweight and gestational diabetes mellitus. Gynecol Endocrinol 22:343-349

7. Lowe LP, Metzger BE, Dyer AR et al (2012) Hyperglycemia and Adverse Pregnancy Outcome (HAPO) study: associations of maternal $\mathrm{A} 1 \mathrm{C}$ and glucose with pregnancy outcomes. Diabetes Care 35:574-580

8. Aris IM, Soh SE, Tint MT et al (2014) Effect of maternal glycemia on neonatal adiposity in a multiethnic Asian birth cohort. J Clin Endocrinol Metab 99:240-247

9. Friis CM, Qvigstad E, Paasche Roland MC et al (2013) Newborn body fat: associations with maternal metabolic state and placental size. PLoS One 8:e57467

10. Visiedo F, Bugatto F, Sánchez V, Cózar-Castellano I, Bartha JL, Perdomo G (2013) High glucose levels reduce fatty acid oxidation and increase triglyceride accumulation in human placenta. Am J Physiol Endocrinol Metab 305:E205-E212 